\title{
Formation and evolution of density singularities in hydrodynamics of inelastic gases
}

\author{
Itzhak Fouxon, Baruch Meerson, Michael Assaf, and Eli Livne \\ Racah Institute of Physics, Hebrew University of Jerusalem, Jerusalem 91904, Israel
}

\begin{abstract}
We use ideal hydrodynamics to investigate clustering in a gas of inelastically colliding spheres. The hydrodynamic equations exhibit a finite-time density blowup, where the gas pressure remains finite. The density blowup signals formation of close-packed clusters. The blowup dynamics is universal and describable by exact analytic solutions continuable beyond the blowup time. These solutions show that dilute hydrodynamic equations yield a powerful effective description of a granular gas flow with close-packed clusters, described as finite-mass point-like singularities of the density. This description is similar in spirit to the description of shocks in ordinary ideal gas dynamics.
\end{abstract}

PACS numbers: 45.70.Qj, 47.40.-x

A central problem of non-equilibrium physics is to describe clusters of matter developing from structureless initial states. Examples are numerous and include the large-scale structure of the Universe [1], star formation [2], radiation-driven plasma condensations [3], clustering of inertial particles in turbulent flows [4] and clustering in inelastic gases [5, 6] considered in this Rapid Communication.

Inelastic (or granular) gas, a simple model of granular flow 7], is a dilute assembly of hard spheres which lose energy at collisions. In the simplest version of the model the normal relative velocity of particles is reduced by a constant factor $0 \leq r<1$ upon each collision. At collisions, internal degrees of freedom of the particles absorb, apart from the energy, the entropy of the gas. As a result, inelastic gases exhibit a host of structure forming instabilities, including the famous clustering instability of a freely cooling homogeneous inelastic gas [5, 6, 8, 9, 10, 11, 12, 13]. This instability involves the development of macroscopic solenoidal flow (the shear mode) and potential flow (the clustering mode), the latter causing the formation of clusters of particles where the particle density ultimately approaches the density of close packing of spheres 13]. The physical mechanism behind the initial density buildup is well understood [5, 14]. Collisional energy loss, enhanced by a local density excess, can lead to a gas temperature decrease so strong that the local pressure falls, causing a further gas inflow and density growth. Pressure, therefore, plays a central role in the initial density build up, as corroborated by the linear theory of the clustering instability [5, 6$]$, where the density growth is accompanied by a decreasing in time pressure, having a local spatial minimum. How do non-linear effects change this linear scenario? Efrati et al. [12] and Meerson and Puglisi [13] addressed this question in the geometry of a long and narrow channel which we adopt in this work too. The channel geometry allows only one-dimensional (1D) macroscopic motions and therefore eliminates the shear mode. In the limit of strong instability (see below), Efrati et al. [12] observed numerically a finite-time blow up of the gas density in 1D hydrodynamic equations. Is it possible to establish the density blow up analytically, and does it develop for generic initial conditions? What is the role of pressure in the density blow up? Can hydrodynamics of dilute gas describe the state of the gas with already existing density spikes (interpreted here as close-packed clusters)? These questions are answered in this Rapid Communication.

As clustering only intensifies with inelasticity, it suffices to consider the nearly elastic limit: $1-r \ll 1$. This allows one (see Refs. [7, 15]) to employ hydrodynamics:

$$
\begin{aligned}
& \partial_{t} \rho+\partial_{x}(\rho v)=0, \quad \rho\left(\partial_{t} v+v \partial_{x} v\right)=-\partial_{x}(\rho T), \\
& \partial_{t} T+v \partial_{x} T=-(\gamma-1) T \partial_{x} v-\Lambda \rho T^{3 / 2}
\end{aligned}
$$

where $\rho$ is the gas density, $v$ is the velocity, $T$ is the temperature (the particle mass is put to unity), $p=\rho T$ is the pressure, and $\gamma$ is the adiabatic index $(\gamma=2$ and $5 / 3$ in $2 \mathrm{D}$ and $3 \mathrm{D}$, respectively). Equations (1) and (2) describe a 1D dilute gas flow [16] with bulk energy losses. These are accounted for by the term $\Lambda \rho T^{3 / 2}$ coming from $\left(1-r^{2}\right) T$ (proportional to the inelastic energy loss per collision) times $\rho T^{1 / 2}$ (proportional to the collision rate). Kinetic theory yields $\Lambda=$ $2 \pi^{(d-1) / 2}\left(1-r^{2}\right) \sigma^{d-1} /[d \Gamma(d / 2)]$, where $d>1$ is the space dimension, and $\sigma$ is the particles diameter, see e.g. [9]. The omission of viscous and heat conduction terms in Eqs. (11) and (2) is justified in sufficiently long systems as, during its linear stage, the clustering instability produces, at $1-r \ll 1$, effective initial conditions with the Reynolds number $R e \gg 1$ [5, 6, 17].

As in other 1D compressible flows, it is convenient to go over from the Eulerian coordinate $x$ to the Lagrangian mass coordinate $m(x, t)=\int_{0}^{x} \rho\left(x^{\prime}, t\right) d x^{\prime}$. Here $m(x, t)$ measures the mass content between the origin [19] and point $x$. This renders a simpler form of the equations:

$$
\begin{aligned}
& \partial_{t}(1 / \rho)=\partial_{m} v, \quad \partial_{t} v=-\partial_{m} p \\
& \partial_{t} p=-\gamma p \rho \partial_{m} v-\Lambda p^{3 / 2} \rho^{1 / 2}
\end{aligned}
$$

Importantly, the cooling coefficient $\Lambda$ can be eliminated from Eqs. (3) owing to the following scaling property: $\rho(m, t)=\rho_{*}\left(\Lambda m / \Lambda_{*}, \Lambda t / \Lambda_{*}\right), \quad v(m, t)=$ $v_{*}\left(\Lambda m / \Lambda_{*}, \Lambda t / \Lambda_{*}\right)$ and $p(m, t)=p_{*}\left(\Lambda m / \Lambda_{*}, \Lambda t / \Lambda_{*}\right)$, 


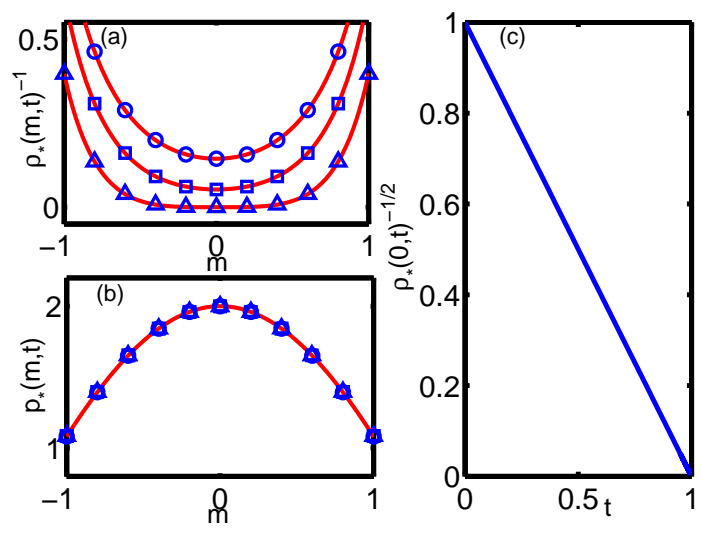

FIG. 1: The rescaled inverse density (a) and pressure (b) at $t=0.62$ (circles), 0.77 (squares) and 0.99 (triangles). The solid lines show the analytical solution (5). (c): the inverse square root of the density at the singularity point $m=0$.

where $u_{*}, v_{*}$ and $p_{*}$ solve Eqs. (3) for $\Lambda_{*}=\gamma \sqrt{2}$. Therefore, in most of the following we put $\Lambda=\Lambda_{*}=\gamma \sqrt{2}$.

Motivated by extensive numerical simulations (we used the classic artificial viscosity, staggered grid scheme of von Neumann and Richtmyer, see Ref. [18]), we found a family of exact time-dependent analytical solutions of Eqs. (3), for which an initially smooth density profile blows up in a finite time $t=t_{c}$. A distinct feature of these solutions is that the fluid elements have a constant in time acceleration, so $v(m, t)$ depends on time linearly, while $p(m, t)$ is time-independent. These solutions are:

$$
\begin{gathered}
\rho_{*}(m, t)=\frac{\rho_{*}(m, 0)}{\left[1-t \sqrt{A \rho_{*}(m, 0) \cos m}\right]^{2}}, p_{*}=2 A \cos m, \\
v_{*}(m, t)=-2 \int_{0}^{m} \sqrt{\frac{A \cos m^{\prime}}{\rho_{*}\left(m^{\prime}, 0\right)}} d m^{\prime}+2 A t \sin m,
\end{gathered}
$$

where $\rho_{*}(m, 0)$ is the initial density (an arbitrary function) [19], and $A>0$ is an arbitrary constant. If $\rho_{*}(m, 0)$ has a maximum at $m=0$, then $\rho_{*}(m, t)$ blows up as $\left(t_{c}-t\right)^{-2}$ at $m=0$ and $t=t_{c}=\left[A \rho_{*}(0,0)\right]^{-1 / 2}$. As $p$ must be non-negative, these solutions hold only at $|m| \leq \pi / 2$, implying a finite mass of the gas $m=\pi$. Depending on the behavior of $\rho_{*}(m, 0)$ near $|m|=\pi / 2$, this finite mass can be distributed over either an infinite, or a finite $x$-interval. A simple example of the former,

$$
\begin{gathered}
\rho_{*}(m, t)=\rho_{0} \cos m\left(1-t \sqrt{A \rho_{0}} \cos m\right)^{-2}, p_{*}=2 A \cos m, \\
v_{*}(m, t)=-2\left(A / \rho_{0}\right)^{1 / 2} m+2 A t \sin m,
\end{gathered}
$$

exhibits a density blow up at $m=0$ and $t_{c}=\left(A \rho_{0}\right)^{-1 / 2}$. We confirmed this solution numerically which indicates its stability with respect to small perturbations, see Fig. 1.

Another exact solution (details will appear elsewhere [20]) shows that an emerging density blowup may coexist with an "ordinary" gas dynamic shock, and the RankineHugoniot conditions at the shock [16] are obeyed. This solution involves, at $t=0$, a zero-temperature gas with density $\rho_{*}(m, 0)=\rho_{0} \exp (2 \sqrt{\gamma-1} m) \cos ^{3} m$ on the Lagrangian interval $0 \leq m \leq \pi / 2$. The gas moves uniformly, $v_{*}(x, 0)=-v_{0}$, and at $t=0$ hits a rigid wall located at $x=0$. A reflected shock propagates into the gas $(x>0)$, leaving behind a flow of the form (4) with $\rho_{*}(m, 0)=\rho_{0}(\gamma+1)[\Phi(m) \sqrt{\cos m}+$ $\Phi^{\prime}(m) \sqrt{(\gamma-1) \cos m}^{-2}$ and $A=\rho_{0} v_{0}^{2}(\gamma+1) / 4$. The function $\Phi(m)=\int_{0}^{m} \exp \left[-m^{\prime} \sqrt{\gamma-1}\right] d m^{\prime} / \cos ^{2} m^{\prime}$ determines the Lagrangian shock coordinate $m_{s}(t)$ implicitly by $t\left(m_{s}\right)=2 \Phi\left(m_{s}\right) /\left[\rho_{0} v_{0}(\gamma+1)\right]$. The collisional energy loss slows down the gas reflection from the wall, producing a density blowup at $m=0$ and $t=t_{c}=$ $2 \sqrt{\gamma-1} /\left[\rho_{0} v_{0}(\gamma+1)\right]$.

These exact solutions (note that they are not selfsimilar) describe a density blowup $\sim\left(t_{c}-t\right)^{-2}$ at any $\Lambda>0$. As $p$ is finite (and non-zero), $T$ vanishes at the singularity. The gas velocity is finite, while its $x$ derivative diverges as $\left(t_{c}-t\right)^{-1}$. In the Eulerian coordinate $x$ the blowup of $\rho$ occurs on a shrinking interval $\Delta(t) \sim\left(t_{c}-t\right)^{5 / 2}$, while $\rho_{*} \sim x^{-4 / 5}$ and does not depend on time at $\Delta(t) \ll|x| \ll 1$. It is instructive to compare this new singularity with the well-known free-flow singularity [21], where the density blows up as $\left(t_{c}-t\right)^{-1}$, the shrinking interval is $\Delta \sim\left(t_{c}-t\right)^{3 / 2}$, and the power law tail of the density at singularity is $\sim x^{-2 / 3}$. For the freeflow singularity it is the Lagrangian velocity, rather than the Lagrangian acceleration, that is constant in time.

Our numerical simulations with Eqs. (11) and (2) showed that, for generic initial conditions, a finite-time density blow up always occurs 22]. Remarkably, the local flow structure near the singularity always coincides with that exhibited by the special solutions (4). One series of simulations used the initial condition $\rho(m, 0)=\rho_{0}$, $T(m, 0)=T_{0}$ and $v(m, 0)=-v_{0} \tanh \left[m /\left(\rho_{0} l\right)\right]$ at different values of two governing scaled parameters: the Mach number $M=v_{0} / T_{0}^{1 / 2}$ and the cooling coefficient rescaled by $\rho_{0} l / M$ (and denoted by $\Lambda$ with no ambiguity). The peak density $\rho(0, t)$ exhibits a finite-time blow up, see Fig. 2, even at a relatively small $\Lambda=0.5$, whereas $p$ remains finite. Moreover, $\rho^{-1 / 2}(0, t)$ goes to zero linearly as $t \rightarrow t_{c}$. We observed these properties to hold at all $\Lambda$ and $M$ for which a high density is reached within a reasonable computation time (generally $t_{c} \rightarrow \infty$ as $\Lambda \rightarrow 0$ ). Figure 3 shows that $-\partial_{m}^{2} \ln p$ at $m=0$ and $t \simeq t_{c}$ vs. $\Lambda$ is equal to $\Lambda^{2} / 8$ : the prediction of Eq. (4) with the restored $\Lambda$-dependence at $\gamma=2$. The same features (the density blowup and the universal flow structure near the singularity) are also observed when starting from a small-amplitude sinusoidal density or velocity perturbation around the homogeneous state. These results leave little doubt that the asymptotic behavior of the hydrodynamic fields near the singularity is described by Eqs. (4).

All the above implies that the non-linear evolution of the clustering instability, as described by the ideal hydrodynamics of a dilute inelastic gas, brings about an 

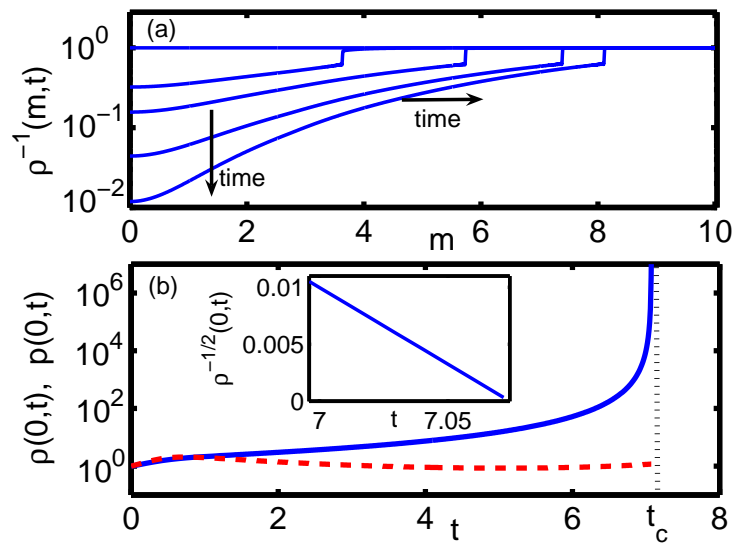

FIG. 2: The inverse density profiles at times 0, 2.06, 3.76, 5.36 , and 6.26 (a) and the evolution of the density (the solid line) and pressure (the dashed line) at the singularity point $m=0$ (b). The inset depicts $\rho^{-1 / 2}(m=0, t)$ close to $t=t_{c}$. The parameters are $\gamma=2, M=1$ and $\Lambda=0.5$.

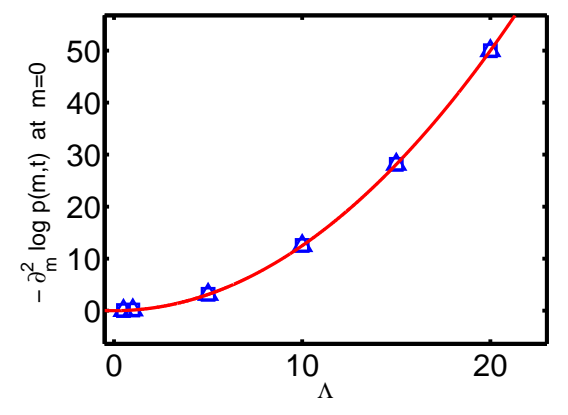

FIG. 3: $-\partial_{m}^{2} \ln p(m, t)$ at $m=0$ and $t \simeq t_{c}$ vs. $\Lambda$ for $M=1$ (squares) and $M=10$ (triangles). The solid line shows $\Lambda^{2} / 8$.

infinite density. In sharp contrast with the linear stage of clustering instability [5, [6] , the gas pressure develops a local maximum at the point of the maximum density, and becomes time-independent in the Lagrangian frame. Obviously, to produce a density blowup, the flow must develop a region with $\partial_{x} v<0$. A negative shear at $t=0$ is necessary and sufficient for a blowup in the limit of $\Lambda \gg 1$, other parameters being fixed. This is because the pressure drops almost instantaneously there, resulting in a free flow and a density singularity development in the regions where $\partial_{x} v<0[12$, 21]. As the velocity profile steepens, however, the compressional heating builds up and arrests the temperature drop, while the pressure again becomes important. The flow, having already developed a high density peak, rearranges, and the blowup develops according to the new (non-free-flow) scenario. The rearrangement occurs within a time interval shrinking to zero as $\Lambda \rightarrow \infty$. At the end of this interval the temperature dives to zero. So, as $\Lambda \rightarrow \infty$, one has $t_{c} \rightarrow\left|\min \partial_{x} v(x, 0)\right|^{-1}$, the blowup time of the free flow [21]. This explains the results of Ref. [12] in a new light. Furthermore, if excluded volume effects are taken into account then, for sufficiently large $\Lambda$, the singularity remains of the free-flow type all the way until the closepacking density is reached 13]. Still later dynamics is describable then by the Burgers equation [13, 23].

Now we return to our special solutions (44). Remarkably, the dilute-gas equations (1) and (2) allow for an effective description of the states of inelastic gas with infinite density spikes. As differential equations of gas dynamics is indeterminate on a spike, one needs to return to the integral form of the mass, momentum and energy balance. Let us continue the solution (4) beyond $t=t_{c}$. We will describe this procedure for $m \geq 0$ [19]. Assume that $\rho_{*}(m, t=0)$ has a maximum at $m=0$ and is monotone decreasing at $m>0$, vanishing at $m=\pi / 2$. Then at $t \geq t_{c}$ there is a point $0 \leq m_{*}(t)<\pi / 2$, implicitly defined by $1=t \sqrt{A \rho_{*}\left(m_{*}(t), 0\right) \cos m_{*}(t)}$, such that $\rho_{*}\left[m_{*}(t), t\right]$, as prescribed by Eq. (4), is infinite. Now, at $t<t_{c}$ we formally put $m_{*}(t)=0$ so $m_{*}(t)$ is continuous at $t=t_{c}$. Here is the solution in Eulerian coordinates, $\hat{\rho}(x, t), \hat{p}(x, t)$ and $\hat{v}(x, t)$, which holds at any $t>0$ :

$$
\hat{\rho}(x, t)=2 m_{*}(t) \delta(x)+\rho_{*}(x, t), \quad \hat{p}(x, t)=p_{*}(x, t),
$$

where $\rho_{*}(x, t)$ and $p_{*}(x, t)$ are given implicitly at $x \geq 0$ by the first line of Eqs. (4) and $x(m, t)=$ $\int_{m_{*}(t)}^{m^{2}} d m^{\prime} / \rho_{*}\left(m^{\prime}, t\right)$. The gas velocity is

$$
\hat{v}=-2 \int_{m_{*}(t)}^{m} \sqrt{\frac{A \cos m^{\prime}}{\rho_{*}\left(m^{\prime}, 0\right)}} d m^{\prime}+2 A t\left[\sin m-\sin m_{*}(t)\right]
$$

with the same $x(m, t)$. We observe that, at $t>t_{c}$, the solution includes a finite mass $2 m_{*}(t)$ concentrated at the origin. It is easy to see that Eqs. (6) and (7) solve Eqs. (3) in the $m$-coordinate at $m>m_{*}(t)$ [but not at $\left.0 \leq m<m_{*}(t)\right]$. As a result, they solve Eqs. (11) and (2) in the $x$-coordinate at $x>0$. It is left to verify that the integral form of Eqs. (11) and (2) holds at $x=0$. The mass flux $\rho v$, which is an odd function of $x$, has a discontinuity at $x=0$ that produces the condition $\dot{m}_{*}=$ $-\lim _{x \rightarrow+0} \rho(x, t) v(x, t)=-\lim _{m \rightarrow+m_{*}(t)} \rho(m, t) v(m, t)$ verifiable using L'Hopital's rule. The integral forms of the momentum and energy equations demand continuity of the momentum and energy fluxes at $x=0$ [for example, $\lim _{\epsilon \rightarrow 0} \int_{-\epsilon}^{\epsilon} \rho^{2} T^{3 / 2} d x=\lim _{\epsilon \rightarrow 0} \int_{-\epsilon}^{\epsilon} \rho p T^{1 / 2} d x=0$ by virtue of $T(x=0, t)=0$ and finiteness of $p(x=0, t)]$. It can be checked that Eqs. (6) and (7) obey these demands. As $t \rightarrow \infty$, all the gas mass accumulates at $x=0$. The same continuation procedure, applied to our timedependent solution with the shock, gives an example of a flow where a density spike and a shock are both present.

According to molecular dynamic (MD) simulations 13], the density buildup in the clusters is arrested only when the close-packing density is reached. An additional exact solution of Eqs. (11) and (2) establishes a simple relation between the density spikes and the close-packed 
clusters. This solution involves a constant gas flux from infinity. In a moving frame the problem is equivalent to that of a piston entering, with speed $v_{0}$, into a cold gas at rest. The latter problem was previously addressed by Goldshtein et al. [24]. Using an empiric equation of state where the pressure diverges at the close-packing density, they found that, at long times, the flow has three regions. First, there is a close-packed cluster, in contact with the piston; the cluster size grows linearly in time. It is followed by a region with non-trivial density, temperature and velocity profiles, separated from the gas at rest by a shock. The shock speed tends to $v_{0}$ in the dilute limit 24]. Importantly, this flow is describable by the dilute equations (11) and (2). In the piston reference frame we obtain $\rho=M(t) \delta(x)+\rho_{s t}(x), v=v_{s t}(x)$ and $T=T_{s t}(x)$, where $\rho_{s t}(x), v_{s t}(x)$ and $T_{s t}(x)$ form unique steady state solution of Eqs. (11) and (2) obeying the boundary conditions $\rho(x=+\infty, t)=\rho_{0}, v(x=+\infty, t)=-v_{0}<0$ and $T(x=+\infty, t)=0$. In this description the close-packed cluster of Ref. 24 becomes a single point $x=0$ which contributes the term $M(t) \delta(x)$ to the gas density. The mass $M(t)$ is coming from infinity, $\dot{M}=\rho_{0} v_{0}$, and stored at $x=0$. The shock is steady at $x=L>0$, while at $x>L$ the gas is at rest. In the region $0<x<L$ the mass and momentum fluxes are constant in space, so one can express $v_{s t}(x)$ and $T_{s t}(x)$ via $\rho_{s t}(x)$. The latter is given implicitly by

$$
x=\frac{1}{\Lambda \rho_{0}}\left\{(\gamma+3) \arcsin \sqrt{u}-\frac{\sqrt{u}[\gamma+3-(\gamma+1) u]}{\sqrt{1-u}}\right\},
$$

where $u=\rho_{0} / \rho_{\text {st }}$, and the $\Lambda$-dependence is restored. The piston-to-shock distance is

$$
L=\frac{1}{\Lambda \rho_{0}}\left[(\gamma+3) \arcsin \sqrt{\frac{\gamma-1}{\gamma+1}}-\sqrt{8(\gamma-1)}\right] .
$$

This characteristic length scale behaves as $\Lambda^{-1}$, so it is very small at $\Lambda \gg 1$. The same feature holds for all solutions that we have presented here.

In summary, ideal hydrodynamic equations for a freely cooling dilute inelastic gas describe the formation of density singularities in a finite time. What becomes of the singularities in microscopic theory? When the local gas density approaches the close-packing density, the singularities must get regularized and give way to (finite-density and finite-size) close-packed particle clusters [13, 17]. One way to put the regularization into the continuum theory is to adopt an equation of state that diverges at the close-packing density [24]. An alternative, suggested in this Rapid Communication, does not demand any regularization. It is similar in spirit to the ideal gas dynamic treatment of shock fronts (which in fact have a finite width) as discontinuities, that is point singularities of the first derivatives of the hydrodynamic fields [16]. As we have shown here, this alternative yields a powerful effective description in terms of smooth flow regions separated by point singularities: either "ordinary" shocks, or density spikes (close-packed clusters). It will be interesting to apply this description to driven flows of granular gases where theory can be compared with experiment. Finally, a direct comparison of our exact solutions with MD simulations is presently under way.

We thank G. Falkovich and P.V. Sasorov for useful comments. This work was supported by the Israel Science Foundation (grant No. 107/05) and by the GermanIsrael Foundation for Scientific Research and Development (Grant I-795-166.10/2003).

[1] P. J. E. Peebles, Large-Scale Structure of the Universe, (Princeton University Press, Princeton, 1980).

[2] F. Palla and G. Meynet, Physics of Star Formation in Galaxies (Springer, Berlin, 2002).

[3] B. Meerson, Rev. Mod. Phys. 68, 215 (1996).

[4] E. Balkovsky, G. Falkovich, and A. Fouxon, Phys. Rev. Lett. 86, 2790 (2001).

[5] I. Goldhirsch and G. Zanetti, Phys. Rev. Lett. 70, 1619 (1993).

[6] S. McNamara, Phys. Fluids A 5, 3056 (1993); S. McNamara and W.R. Young, Phys. Rev. E 53, 5089 (1996).

[7] N.V. Brilliantov and T. Pöschel, Kinetic Theory of Granular Gases (Oxford University Press, Oxford, 2004).

[8] R. Brito and M. H. Ernst, Europhys. Lett. 43, 497 (1998).

[9] J.J. Brey, M.J. Ruiz-Montero, and D. Cubero, Phys. Rev. E 60, 3150 (1999).

[10] S. Luding and H. J. Herrmann, Chaos 9, 673 (1999).

[11] X.B. Nie, E. Ben-Naim, and S.Y. Chen, Phys. Rev. Lett. 89, 204301 (2002).

[12] E. Efrati, E. Livne, and B. Meerson, Phys. Rev. Lett. 94, 088001 (2005).

[13] B. Meerson and A. Puglisi, Europhys. Lett. 70, 478 (2005).

[14] G.B. Field, Astrophys. J. 142, 531 (1965).

[15] I. Goldhirsch, Annu. Rev. Fluid Mech. 35, 267 (2003).

[16] L. D. Landau and E. M. Lifshitz, Fluid Mechanics (Pergamon Press, Oxford, 1987); A.J. Chorin and J.E. Marsden, A Mathematical Introduction to Fluid Mechanics (Springer, Berlin, 2000).

[17] Numerical simulations suggest that an account of viscosity and heat conduction may not arrest the density singularities [12].

[18] R.D. Richtmyer and K.W. Morton, Difference Methods for Initial Value Problems (Interscience, New York, 1967).

[19] We assume that $p(x, t)$ and $\rho(x, t)$ are even functions of $x$, while $v(x, t)$ is odd so that $v(x=0, t)=v(m=0, t)=0$.

[20] I. Fouxon, B. Meerson, M. Assaf, and E. Livne, arXiv:0704.0084 [cond-mat.soft].

[21] G.B. Whitham, Linear and Nonlinear Waves (Wiley, New York, 1974), Chapter 2.

[22] The numerical simulations had to be terminated at a very high but finite density.

[23] E. Ben-Naim, S.Y. Chen, G.D. Doolen, and S. Redner, Phys. Rev. Lett. 83, 4069 (1999).

[24] A. Goldshtein, M. Shapiro, and C. Gutfinger, J. Fluid. Mech. 316, 29 (1996). 